\title{
QCD factorization for forward hadron scattering at high energies
}

\author{
B.I. Ermolaev \\ Ioffe Physico-Technical Institute, 194021 St.Petersburg, Russia \\ M. Greco \\ Department of Physics and INFN, University Roma Tre, Rome, Italy \\ S.I. Troyan \\ St.Petersburg Institute of Nuclear Physics, 188300 Gatchina, Russia
}

\begin{abstract}
We consider the QCD factorization of DIS structure functions at small $x$ and amplitudes of $2 \rightarrow 2$ -hadronic forward scattering at high energy. We show that both collinear and $k_{T}$-factorization for these processes can be obtained approximately as reductions of a more general (totally unintegrated) form of the factorization. The requirement of ultraviolet and infrared stability of the factorization convolutions allows us to obtain restrictions on the fits for the parton distributions in $k_{T^{-}}$and collinear factorization.
\end{abstract}

PACS numbers: 12.38.Cy 


\section{INTRODUCTION}

QCD factorization is the fundamental concept to provide theoretical grounds for applying Perturbative QCD to the description of hadronic reactions. According to factorization, any scattering amplitude $A$ in QCD can be represented as a convolution of perturbative (E) and non-perturbative (T) contributions. In particular, the representation of the amplitude $A$ for the elastic Compton scattering

$$
\gamma(q)+h(p) \rightarrow \gamma(q)+h(p)
$$

off a hadron target is

$$
A=\sum_{r} E_{r} \otimes T_{r}
$$

where $r$ denotes the type of intermediate partons in the convolutions: quarks or gluons. Similarly, the amplitudes $A_{h}$ of $2 \rightarrow 2$-hadronic reactions

$$
h_{1}\left(p_{1}\right)+h_{2}\left(p_{2}\right) \rightarrow h_{1}^{\prime}\left(p_{1}^{\prime}\right)+h_{2}^{\prime}\left(p_{2}^{\prime}\right)
$$

can be represented in a general form through more involved convolutions:

$$
A_{h}=\sum_{r r^{\prime}} \widetilde{T}_{r} \otimes A_{r r^{\prime}}^{(p e r t)} \otimes T_{r^{\prime}}
$$

where $T$ and $\widetilde{T}$ are parton distributions and $A_{r r^{\prime}}^{(p e r t)}$ is the perturbative amplitude, with the purely gluonic contribution $A_{g g}^{(p e r t)}$ dominating in forward kinematics at very high energies.

There are two kinds of QCD factorization in the literature: Collinear factorization[1, 2] and $k_{T^{-}}$factorization[3]. For instance, the DIS structure functions $f\left(x, Q^{2}\right)$ are respectively represented in those factorizations as follows:

$$
f\left(x, Q^{2}\right)=\int_{x}^{1} \frac{d \beta}{\beta} f^{(\text {pert })}\left(x / \beta, Q^{2} / \mu^{2}\right) \phi\left(\beta, \mu^{2}\right)
$$

and

$$
f\left(x, Q^{2}\right)=\int_{x}^{1} \frac{d \beta}{\beta} \int \frac{d k_{\perp}^{2}}{k_{\perp}^{2}} f^{(p e r t)}\left(x / \beta, Q^{2} / k_{\perp}^{2}\right) \Phi\left(\beta, k_{\perp}^{2}\right)
$$

where $f^{(\text {pert })}$ stands for the perturbative components of the structure functions both in collinear and $k_{T}$-factorization while $\phi$ and $\Phi$ denote the involved parton distributions. In the present paper we discuss only general features of these perturbative components, so throughout the paper we will keep for them the same generic notation $f^{(p e r t)}$ in both collinear and $k_{T}$-factorization. In contrast, we are going to discuss details of the involved parton distributions, so, in order to avoid any misunderstanding, we have used in Eqs. (56) different notations for the parton distributions in collinear and $k_{T}$-factorization, $\phi$ and $\Phi$, respectively. Besides collinear and $k_{T^{-}}$factorization, we will introduce in Eq. (13) a more general factorization which we call basic factorization. This factorization involves new, totally unintegrated parton distributions which we will denote $\Psi$. The parameter $\mu$ in Eq. (5) denotes the factorization scale of collinear factorization. Originally, this scale parameter was introduced a kind of border between the perturbative and non-perturbative domains of QCD. In addition, it plays the role of the cut-off for the infrared-divergent perturbative contributions. Also, it is often associated with the starting point of $Q^{2}$-evolution. Collinear factorization Eq. (2) treats the intermediate partons as nearly on-shell ones. In particular, this takes place in DGLAP [4] and its generalizations (see e.g. the overview [5]) to the small- $x$ region. The collinear factorization cannot be used when the perturbative contributions to Eq. (2) are calculated using BFKL[6], where the external gluons are kept essentially off-shell. In order to embrace this case, collinear factorization was replaced by $k_{T}$-factorization. $k_{T^{-}}$and collinear factorization, being introduced for such different motivations, look unrelated to each other. In Ref. 7] we showed that both factorizations for the DIS structure functions can be derived from a more general factorization, which we addressed as the Basic form of QCD factorization, where the convolutions are totally unintegrated. 
In the present paper we continue to investigate the relations between $k_{T^{-}}$and collinear factorization in more detail than was done in Ref. [7], paying more attention to the gauge invariance of the amplitudes involved and considering a more complicated dependence of the parton distributions on the transverse momenta. We first consider DIS structure functions and then generalize the results obtained for them to the more intricate convolutions describing factorization of the amplitudes of $2 \rightarrow 2$-hadron scattering. Throughout the paper we focus on the high-energy (small- $x$ ) domain. Our paper is organized as follows: In Sect. II we consider the derivation of the collinear and $k_{T}$-factorization for the amplitudes of forward Compton scattering off a hadron target and then proceed to the amplitudes of the forward hadron scattering in Sect. [II] In Sect.[V] we compare the form of collinear factorization obtained in Sects. [II [II with the conventional form of this factorization and relate these two forms. In Sect. $\mathrm{V}$ we discuss the ultraviolet behavior of the totally unintegrated parton distributions and formulate the requirements for the factorization convolutions to be UV-stable; applying these requirements to the standard fits in Sect. VI, we argue against the use the singular factors $x^{-a}$ in the fits. We summarize our results in Sect. VII]

\section{FACTORIZATION FOR FORWARD COMPTON SCATTERING}

The scattering amplitude $A_{\mu \nu}(p, q)$ of forward Compton scattering off a hadron target is depicted in Fig. 1

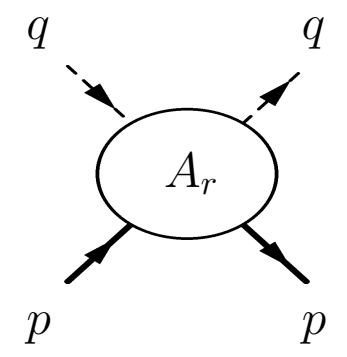

FIG. 1. Forward Compton scattering off a hadron target.

where the blob signifies the presence of perturbative and non-perturbative QCD contributions. We have used the standard notations: $p$ stands for the hadron momentum and $q\left(-q^{2}=Q^{2}\right)$ is the virtual photon momentum. In what follows we omit dependence of $A_{\mu \nu}$ on the hadron mass and spin. Due to the Optical theorem, the imaginary part $\Im A_{\mu \nu}$ is proportional to the hadronic tensor $W_{\mu \nu}$. Using the standard projection operators, $A_{\mu \nu}$ can be expanded into a set of invariant amplitudes $A_{r}$, every DIS structure function $f_{r}$ being expressed through a certain $A_{r}$ :

$$
f_{r}=\frac{1}{\pi} \Im A_{r}
$$

The next step is to represent $A_{r}$ in a factorized form as an infinite set of $t$-channel convolutions, each with a certain number of the intermediate $t$-channel partons (quarks and gluons) as shown in Fig. 2, Each of the blobs in Fig. 2 can contain both perturbative and non-perturbative contributions. In the present paper we consider only the simplest convolutions (a) and (b) in Fig. 2 with two-quark and two-gluon intermediate states respectively, denoting them by $A_{r}^{(q)}$ and $A_{r}^{(g)}$ respectively. In other words, we assume that

$$
A_{r} \approx A_{r}^{(q)}+A_{r}^{(g)}
$$

Let us notice that our assumption is in full agreement with the conventional approach to factorization. On the other hand, neglecting the contributions with multi-parton intermediate states could rise questions about the gauge invariance of $A_{r}^{(q)}$ and $A_{r}^{(g)}$. We discuss the gauge invariance of them in the present Sect. We will focus on discussing $A_{r}^{(q)}$ while the convolution $A_{r}^{(g)}$ with two-gluon intermediate state can be studied quite similarly (see Ref. [7] for detail). In the analytical form, $A_{r}^{(q)}$ is 


$$
A_{r}^{(q)}(p, q)=\int \frac{d^{4} k}{(2 \pi)^{4}} \frac{\hat{k} \tilde{A}_{r}(q, k) \hat{k} T_{r}(p, k)}{k^{2} k^{2}}
$$

where $\tilde{A}_{r}\left(T_{r}\right)$ denotes the upper (lowest) blob in Fig. 2(a) and $k$ stands for the intermediate quark momentum.

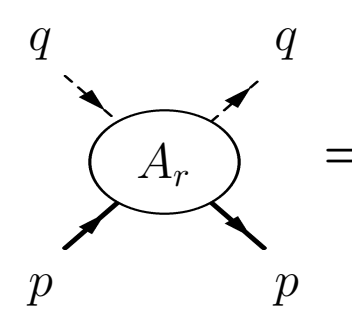

$p$

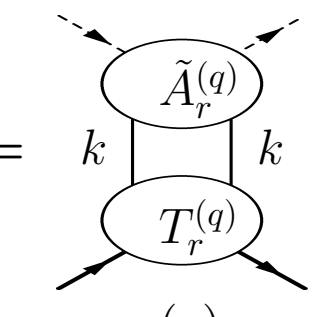

(a)

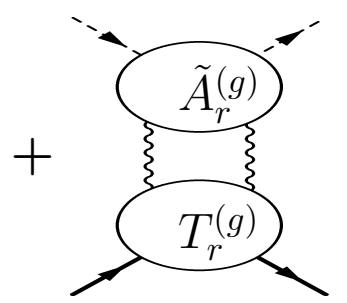

(b)

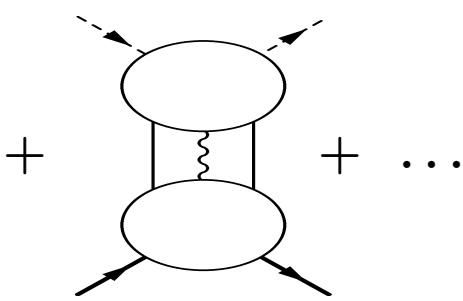

(c)

FIG. 2. Representation of Compton amplitude through convolutions.

Each of $\tilde{A}_{r}$ and $T_{r}$ can contain both perturbative and non-perturbative contributions, so the factorized form of $A_{r}$ in Eq. (9) does not correspond to the concept of QCD factorization where the perturbative and non-perturbative contributions should be separated. Because of this reason we refer to Eq. (9) as the Primordial Convolution. The integration in Eq. (9) runs over the whole phase space and includes both the ultraviolet (UV) and infrared (IR) regions. The perturbative contributions in $\tilde{A}_{r}$ are IR-divergent and must be regulated. In the first place, there is the IR-divergent power contribution $2 p k / k^{2}$. Such a contribution appears in one of the amplitudes $\tilde{A}_{r}$ with vacuum quantum numbers in the $t$-channel. Throughout the paper we will refer to such an amplitude as the singlet amplitude and denote it by $A_{S}$, with the upper blob $\tilde{A}_{S}$ and the lower blob $T_{S}$, omitting the superscript $q$. Through the Optical theorem $A_{S}$ is related to the singlet DIS structure function $F_{1}$. We will refer to all other amplitude as non-singlet ones and generically denote them $A_{N S}$, with the upper and lower blobs $\tilde{A}_{N S}$ and $T_{N S}$ respectively. We stress that some of such non-singlet amplitudes are related to the flavor singlet structure functions (for example, $F_{2}, g_{1}^{S}$, etc). Besides the power IR-dependent term, there are IR-divergent logarithmic contributions $\sim \ln ^{n}\left(2 p k / k^{2}\right)$ and $\ln ^{n}\left(Q^{2} / k^{2}\right)$. Such contributions exist in the singlet and non-singlet amplitudes. It was shown in Ref. 7] that in order to regulate the IR divergences in $A_{S}$ and $A_{N S}$ without explicitly cutting off the region of small $k^{2}$ from the integration region in Eq. (9), the amplitudes $T_{S}, T_{N S}$ should obey

$$
T_{N S} \sim\left(k^{2}\right)^{\gamma}, \quad T_{S} \sim\left(k^{2}\right)^{1+\gamma}
$$

at small $k^{2}$, with $\gamma>0$. It is convenient to rewrite Eq. (9) in terms of the standard Sudakov variables $[8]$ :

$$
k=-\alpha(q+x p)+\beta\left(p-x^{\prime} q\right)+k_{\perp},
$$

where $x=-q^{2} / w, x^{\prime}=p^{2} / w$ and $w=2 p q$. Doing so, we arrive at

$$
A_{r}^{(q)}(p, q)=\frac{w}{32 \pi^{3}} \int d \alpha d \beta d k_{\perp}^{2} \frac{\hat{k} \tilde{A}_{r}(q, k) \hat{k} T_{r}(p, k)}{\left(w \alpha \beta+k_{\perp}^{2}\right)^{2}}
$$

where $r=S, N S$. Now let us consider $A_{r}^{(q)}(p, q)$ in the Born approximation. The Compton amplitude off the quark (the upper blob) in the Born approximation is depicted in Fig. 3 .

The quark amplitudes $A_{S, N S}^{(q)}$ and the gluon amplitudes $A^{(g)}$ in the Born approximation were investigated in detail in Ref. [7], so we omit this in the present paper. Let us stress that the lower blobs $T_{S}$ and $T_{N S}$ in the Born approximation are altogether non-perturbative. As is known, the Born Compton amplitude is gauge-invariant when the quark is on-shell. In Appendix A we demonstrate that gauge invariance for those amplitude is restored in the high-energy region where $w \gg Q^{2}, k^{2}$. 


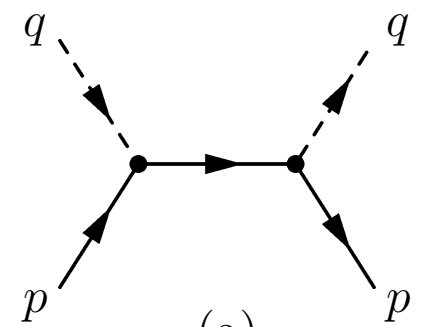

(a)

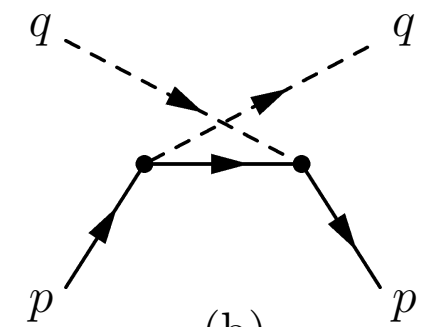

(b)

FIG. 3. The Born contribution to Compton amplitude.

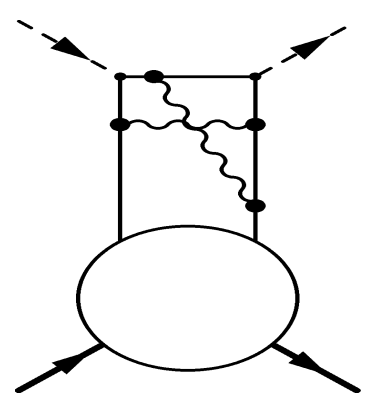

(a)

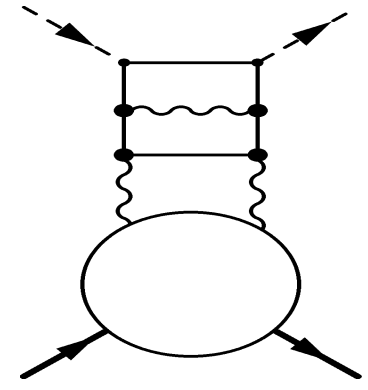

(b)

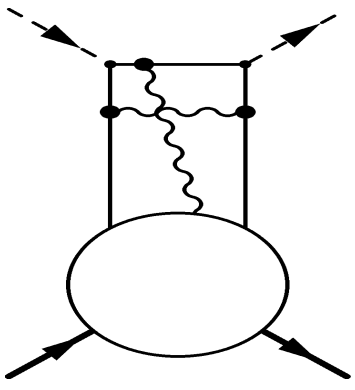

(c)

FIG. 4. Examples of the graphs contributing to Compton amplitude beyond the Born approximation.

Going beyond the Born approximation means adding extra gluon and quark propagators in all possible ways to the Born graphs. Examples of this are shown in Fig. 4.

The resulting graphs can be classified into two groups:

(i) The graphs where extra propagators do not affect the lower blob (for instance the graphs (a) and (b) in Fig. (4). (ii) The graphs where the extra propagators involve the lower blob (graph (c) in Fig. 4).

Obviously, accounting for the whole set of graphs from group (i) leads to the convolution depicted in Fig. 2(a). whereas graphs from the group (ii) form convolutions with more complicated intermediate states. Such graphs cannot be simply neglected because this affects the gauge invariance. However, we demonstrate in Appendix A that gauge invariance is restored at small $x$ even when the group (ii) is neglected. Let us stress that we have arrived at the convolution where the upper blob $A_{r}$ is altogether perturbative and at the same time the lower blob $T_{r}$ is totally non-perturbative.

Applying the Optical theorem to Eq. (12), simplifying the spinor structure and adding the similar gluon contribution (see Ref. 7] for detail) leads to the convolution for the DIS structure functions:

$$
f_{S, N S}\left(x, Q^{2}\right)=\int d \alpha \frac{d \beta}{\beta} \frac{d k_{\perp}^{2}}{\left(w \alpha \beta+k_{\perp}^{2}\right)} f_{S, N S}^{(p e r t)}\left(x / \beta, Q^{2} / k^{2}\right) \Psi_{S, N S}\left(w \alpha, k^{2}\right)
$$

where $f_{N S}^{(\text {pert })}\left(f_{S}^{(\text {pert })}\right)$ is the perturbative component of $f_{N S}\left(f_{S}\right)$ and $\Psi_{N S}=\Im T_{N S}, \Psi_{S}=\Im T_{S}$. In order to regulate the IR-divergences in Eq. (13), these parton distributions should decrease with $k^{2}$ at small $k^{2}$ :

$$
\Psi_{N S} \sim\left(k^{2}\right)^{\gamma}, \quad \Psi_{S} \sim\left(k^{2}\right)^{1+\gamma} .
$$

Eq. (13) represents the structure functions in factorized form, with perturbative and non-perturbative contributions being separated, so this representation authentically corresponds to the concept of QCD factorization. On the other 
hand, this factorization involves three integrations, which distinguishes it from both $k_{T}$-factorization (which involves integration over $\beta$ and $k_{\perp}$ ) and the collinear factorization (involving integration over $\beta$ only). In Ref. [7] we introduced the term "Basic form of factorization" for such totally unintegrated convolutions and will use it throughout the present paper. The basic factorization involves the totally unintegrated parton distributions. In this respect we are close to Ref. [9] but in contrast to that paper, we focus on small- $x$ kinematics and go beyond the Born approximation.

The basic factorization can be approximately reduced to the $k_{T}$-factorization if the restriction

$$
w \alpha \beta \ll k_{\perp}^{2}
$$

is accepted. In this case one can perform the integration over $\alpha$ without dealing with $f^{(p e r t)}$ and arrive at the $k_{T}$ -factorization for $f_{S}, f_{N S}$ :

$$
f_{S, N S}\left(x, Q^{2}\right) \approx \int_{x}^{1} \frac{d \beta}{\beta} \int_{0}^{w} \frac{d k_{\perp}^{2}}{k_{\perp}^{2}} f_{S, N S}^{(p e r t)}\left(x / \beta, Q^{2} / k_{\perp}^{2}\right) \Phi_{S, N S}\left(\beta, k_{\perp}^{2}\right)
$$

$\Phi_{S}$ and $\Phi_{N S}$ being the parton distributions for the $k_{T}$-factorization. They are related to the totally unintegrated parton distributions $\Psi_{S}, \Psi_{N S}$ :

$$
\Phi_{N S}=\int_{s_{2}^{(0)} / w}^{k_{\perp}^{2} / w \beta} d \alpha \Psi_{N S}\left(w \alpha, k^{2}\right), \quad \Phi_{S}=\int_{s_{2}^{(0)} / w}^{k_{\perp}^{2} / w \beta} d \alpha \Psi_{S}\left(w \alpha, k^{2}\right) .
$$

The lowest limit of integrations in Eq. (17) is fixed from the following considerations: the invariant energy $s_{2}=(p-k)^{2}$ of $\Phi_{N S}$ and $\Phi_{S}$ must be positive, so

$$
s_{2}=(p-k)^{2} \approx w \alpha-k_{\perp}^{2}>s_{2}^{(0)}>0 .
$$

In order to regulate the IR divergences in the $k_{T}$-convolutions, these parton distributions should behave (cf. (10)) as

$$
\Phi_{N S} \sim\left(k_{\perp}^{2}\right)^{\gamma}, \quad \Phi_{S} \sim\left(k_{\perp}^{2}\right)^{1+\gamma}
$$

at small $k_{\perp}^{2}$. Obviously, the $k_{T}$-factorization in Eq. (16) authentically coincides with the conventional form of $k_{T^{-}}$ factorization in Eq. (6).

In its turn, $k_{T}$-factorization can also be approximately reduced to collinear factorization. In order to do it, we assume the peaked dependence of the parton distributions on $k_{\perp}^{2}$ as shown in Fig. 5 .

We stress that this hypothesis can be checked by analysis of experimental data. The peaked dependence $\Phi_{S, N S}$ on $k_{\perp}^{2}$ makes it possible to perform the integrations over $k_{\perp}^{2}$ in Eq. (16) without involving the perturbative components $f_{S, N S}^{(p e r t)}$, arriving at the collinear factorization

$$
f_{S, N S}\left(x, Q^{2}\right) \approx \sum_{k} \int_{x}^{1} \frac{d \beta}{\beta} f_{S, N S}^{(\text {pert })}\left(x / \beta, Q^{2} / \mu_{k}^{2}\right) \phi_{S, N S}\left(\beta, \mu_{k}^{2}\right)
$$

with the singlet and non-singlet parton distributions $\phi_{N S}\left(\beta, \mu_{k}^{2}\right)$ and $\phi_{S}\left(\beta, \mu_{k}^{2}\right)$ defined at the factorization scale $\mu_{k}$. They are related to the $k_{T}$-parton distributions in the following way:

$$
\phi_{N S}=\int_{D_{k}} d k_{\perp}^{2} \Phi_{N S}\left(w \alpha, k_{\perp}^{2}\right), \quad \phi_{S}=\int_{D_{k}} d k_{\perp}^{2} \Phi_{S}\left(w \alpha, k_{\perp}^{2}\right)
$$

The integration regions $D_{k}$ in Eq. (21) are located around the positions of the maxima of the parton distributions at $k_{\perp}^{2}=\mu_{k}^{2}$ as shown in Fig. 5. Obviously, Eq. (20) differs in many respects from the conventional expression in Eq. (5) for the DIS structure functions in collinear factorization. We will show how to bring Eq. (20) to the conventional form in Sect. IV. 


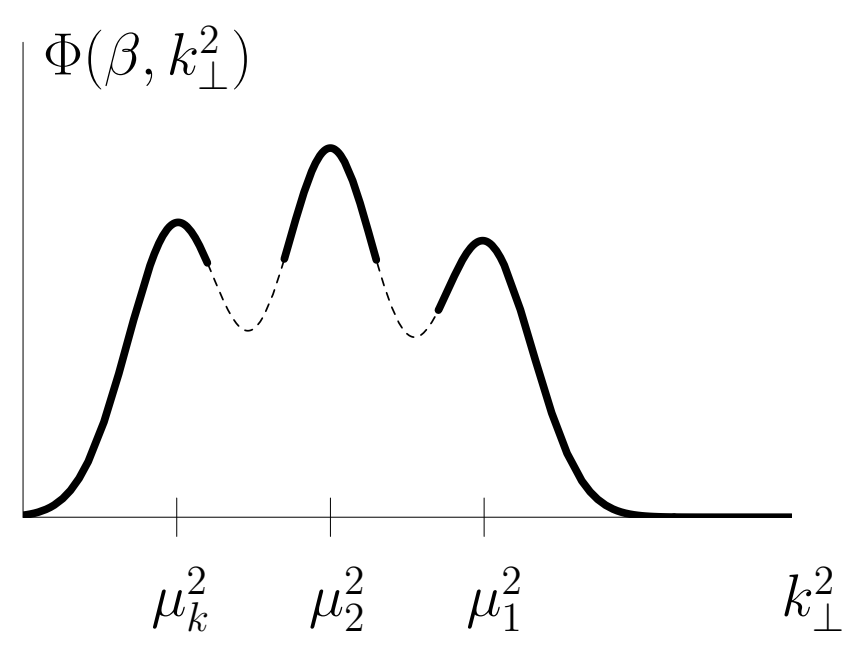

FIG. 5. Assumed dependence of the parton distributions on $k_{\perp}^{2}$.

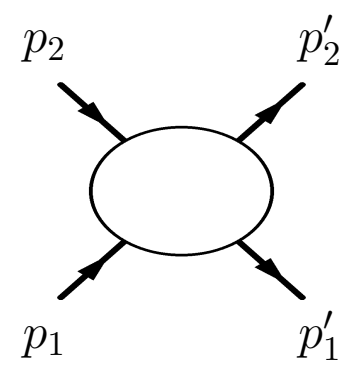

FIG. 6. Forward $2 \rightarrow 2$ scattering of hadrons.

\section{FORWARD SCATTERING OF HADRONS}

Let us consider the $2 \rightarrow 2$-scattering of hadrons (see Eq. (3) $)$ in forward kinematics where $s=\left(p_{1}+p_{2}\right)^{2} \gg t=$ $\left(p_{1}^{\prime}-p_{1}\right)^{2}$. This process is depicted in Fig. 6.

In order to be able to apply Perturbative QCD to this reaction, should be regarded as consisting of the following two sub-processes as depicted in Fig. 7

(i) Each of the colliding hadrons emits one or several partons (the upper and lowest blobs in Fig. 7).

(ii) High-energy interaction of the emitted partons (the middle blob in Fig. 7).

In the present paper we will consider convolutions with two intermediate partons in the $t$-channel (graphs (a) and (b)) only. This approximation allows us to write the scattering amplitude $A_{h}$ of the process of Eq. (3) in the form of the following convolutions (cf. Eq. (2)):

$$
A_{h} \approx \sum_{r r^{\prime}} A_{r r^{\prime}}=\sum_{r r^{\prime}} \widetilde{T}^{\left(r^{\prime}\right)}\left(k_{2}, k_{2}^{\prime}, p_{2}, p_{2}^{\prime}\right) \otimes H^{\left(r^{\prime} r\right)}\left(k_{2}, k_{2}^{\prime}, k_{1}, k_{1}^{\prime}\right) \otimes T^{(r)}\left(p_{1}, p_{1}^{\prime}, k_{1}, k_{1}^{\prime}\right)
$$

where the superscripts $r, r^{\prime}$ refer to the kind of intermediate two-parton states: two-quark or two-gluon states. $T^{(r)}$ and $\widetilde{T}^{\left(r^{\prime}\right)}$ stand for the lowest and upper blobs respectively while $H^{\left(r^{\prime} r\right)}$ denotes the middle blob in Fig. 7 , $k_{1,2}^{\prime}=q+k_{1,2}$, with $q=p_{1}^{\prime}-p_{1}$.

Similarly to the Compton scattering amplitudes considered in Sect. II, the amplitudes $A_{h}$ for hadron scattering can have either vacuum or non-vacuum quantum numbers in the $t$-channel. In the present paper we focus on the singlet 


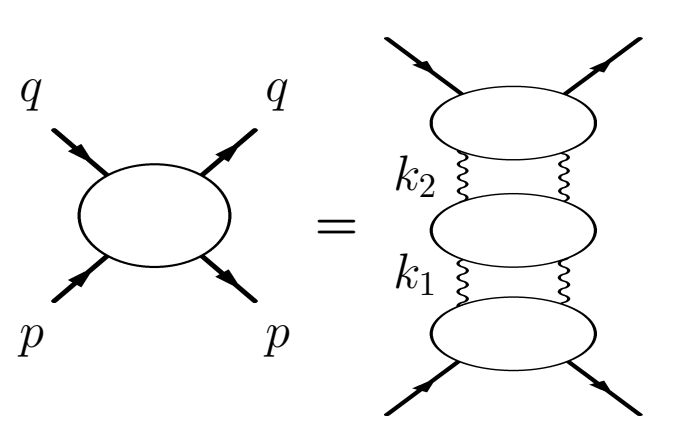

(a)

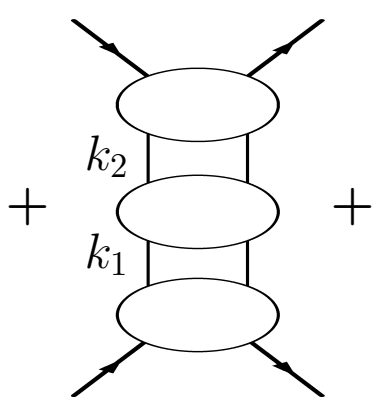

(b)

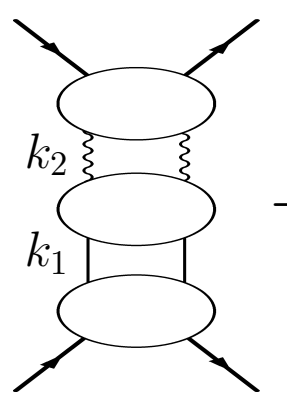

(c)

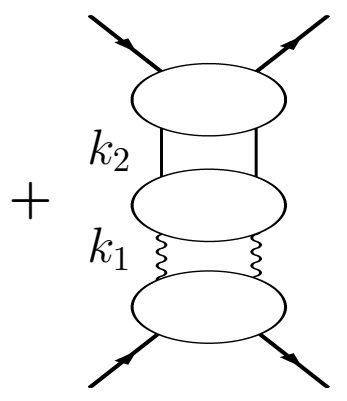

(d)

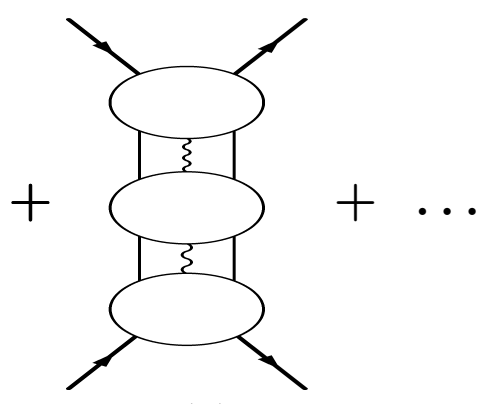

(e)

FIG. 7. Representation of the amplitude $A_{h}$ through convolutions of sub-processes.

amplitudes with the vacuum quantum numbers. Then, in what follows we consider in more detail the amplitude $A_{g g}$ which corresponds to graph (a) in Fig. 7 .

$$
A_{g g}=\int \frac{d^{4} k_{1}}{(2 \pi)^{4}} \frac{d^{4} k_{2}}{(2 \pi)^{4}} \widetilde{T}_{\mu \mu^{\prime}}^{(g)}\left(p_{2}, p_{2}^{\prime}, k_{2}, k^{\prime} 2\right) \frac{1}{k_{2}^{2} k_{2}^{\prime 2}} H_{\mu \mu^{\prime} \lambda \lambda^{\prime}}^{(g g)}\left(k_{2}, k_{1}\right) \frac{1}{k_{1}^{2} k_{1}^{\prime 2}} T_{\lambda \lambda^{\prime}}^{(g)}\left(p_{1}, p_{1}^{\prime}, k_{1}, k_{1}^{\prime}\right),
$$

where the subscripts $\mu, \mu^{\prime}, \lambda, \lambda^{\prime}$ denote the gluon polarizations. In order to account for the leading contributions, we parameterize $T_{\lambda \lambda^{\prime}}^{(g)}$ and $\widetilde{T}_{\mu \mu^{\prime}}^{(g)}$ as follows:

$$
\widetilde{T}_{\mu \mu^{\prime}}^{(g)}=\frac{2 p_{2 \mu} p_{2 \mu^{\prime}}}{s} \widetilde{T}, \quad T_{\lambda \lambda^{\prime}}^{(g)}=\frac{2 p_{1 \lambda} p_{1 \lambda^{\prime}}}{s} T, \quad H=\frac{2 p_{2 \mu} p_{2 \mu^{\prime}}}{s} H_{\mu \mu^{\prime} \lambda \lambda^{\prime}}^{(g g)} \frac{2 p_{1 \lambda} p_{1 \lambda^{\prime}}}{s}
$$

$\widetilde{T}, T, H$ being scalar functions. We have dropped the superscripts here. Using these notations and the Sudakov parametrization of Eq. (11) for $k_{1,2}$, we can rewrite Eq. (23) as follows:

$$
A_{g g}=\int d \alpha_{1,2} d \beta_{1,2} d k_{1,2 \perp}^{2} \widetilde{T}\left(s \beta_{2}, k_{2}^{2}, k_{2}^{\prime 2}\right) \frac{1}{k_{b}^{2}} \frac{s^{\prime}}{k^{2}} M \frac{1}{k_{a}^{\prime 2}} T\left(s \alpha_{1}, k_{1}^{2}, k_{1}^{\prime 2}\right),
$$

with $k_{r}^{2}=-s \alpha_{r} \beta_{r}-k_{r \perp}^{2}$. Let us explain the notations we have introduced in Eq. (25). We have denoted by $s^{\prime}$ the invariant energy for the sub-process of gluon $2 \rightarrow 2$-scattering: $s^{\prime}=\left(k_{1}-k_{2}\right)^{2}=\left(k_{1}^{\prime}-k_{2}^{\prime}\right)^{2} \approx s \alpha_{2} \beta_{1}$. Throughout the paper we consider the kinematics where

$$
s^{\prime} \gg k_{1}^{2}, k_{2}^{2}, k_{1}^{\prime 2}, k_{2}^{\prime 2} .
$$

Let us denote by $k_{c}$ the momentum with the largest virtuality: $k_{c}^{2}=\max \left[k_{1}^{2}, k_{2}^{2}, k_{1}^{\prime 2}, k_{2}^{\prime 2}\right]$. Then we denote by $k_{b}^{2}$ any of $k_{2}^{2}, k_{2}^{\prime 2}$, providing $b \neq c$. Similarly, $k_{a}^{2}$ is any of $k_{1}^{2}, k_{1}^{\prime 2}$, providing $a \neq c$. Finally, $k^{2}=\min \left[k_{1}^{2}, k_{2}^{2}, k_{1}^{\prime 2}, k_{2}^{\prime 2}\right]$. 
The amplitudes $T, \widetilde{T}$ can include both perturbative and non-perturbative contributions, as can amplitude $M$. The amplitude $M$ replaces the amplitude $H$ when the momenta $k_{a}, k_{b}, k$ are used instead of $k_{1}, k_{2}$. The integration over $k_{1,2}$ in Eq. (25) runs over the whole phase space, so the integration region includes the IR-singularities where any of $k_{1}^{2}, k_{1}^{\prime 2}$ or $k_{2}^{2}, k_{2}^{\prime 2}$ can be equal to zero. Obviously, $k_{2}^{2}$ and $k_{2}^{\prime 2}$ (and $k_{1}^{2}$ and $k_{1}^{\prime 2}$ ) can be small at the same time only when $q=0$, i.e.when $t=0$ and therefore the forward kinematics becomes collinear. This kinematics is the most IR-singular. At $t=0$ Eq. (25) looks as follows:

$$
A_{g g}=\int d \alpha_{1,2} d \beta_{1,2} d k_{1,2 \perp}^{2} \widetilde{T}\left(s \beta_{2}, k_{2}^{2}\right) \frac{1}{k_{2}^{2}}\left(\frac{s^{\prime}}{k^{2}}\right) M \frac{1}{k_{1}^{\prime 2}} T\left(s \alpha_{1}, k_{1}^{2}\right)
$$

where $k^{2}=\min \left[k_{2}^{2}, k_{1}^{2}\right]$.

The perturbative infrared-sensitive contributions in the amplitude $M$ are logarithms: $M=M\left(\ln \left(s^{\prime} / k_{1}^{2}\right), \ln \left(s^{\prime} / k_{2}^{2}\right)\right)$.

On the other hand, the hadron amplitude $A_{g g}$ must be free of IR problems by definition. In order to keep Eq. (27) IR-stable, Amplitudes $T$ and $\widetilde{T}$ should compensate the IR-divergent terms (cf. Eq. (10)):

$$
T \sim\left(k_{1}^{2}\right)^{1+\gamma}, \quad \widetilde{T} \sim\left(k_{2}^{2}\right)^{1+\gamma},
$$

with $\gamma>0$. When $t \neq 0$, the conditions for the IR stability are weaker than in Eq. (28):

$$
T \sim\left(k_{a}^{2}\right)^{\gamma}, \quad \widetilde{T} \sim\left(k_{b}^{2}\right)^{\gamma} .
$$

With the integrations in Eqs. (25|27) IR-stable and the total energy of the gluon scattering high, the amplitude $M$ becomes completely perturbative. When $M$ is calculated in the Born approximation, the amplitude $A_{h}$ is represented in Fig. 8. Let us notice that the Born approximation means that the blobs in Fig. 8 include non-perturbative contributions only.

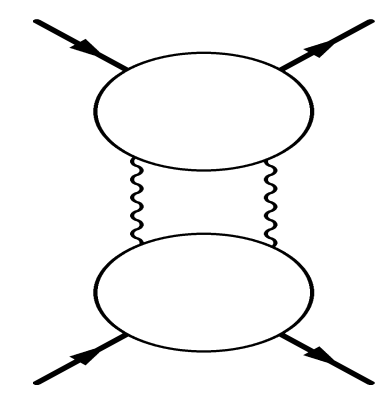

FIG. 8. Convolution for $A_{h}$ in the Born approximation.

Considering $A_{g g}$ beyond the Born approximation corresponds to adding the quark and gluon propagators to the Born graph. In doing so, we account for those graphs which do not involve the upper and lower blobs, like graph (a) in Fig. 9 but avoid including the graphs involving the blobs like graph (b).

Such a procedure keeps the blobs totally non-perturbative. The gauge invariance of the set of retained graphs is proved in Appendix B. As a result, we arrive back at graph (a) depicted in Fig. 7 where, however, the middle blob is perturbative while the upper and lowest blobs are altogether non-perturbative. Therefore, the convolutions in Eqs. (23125) complemented by the IR-regulators of Eqs. (28, 29) represent the hadron scattering amplitude $A_{h}$ in terms of the convolutions of Eqs. (25/27), with the convolutions being in the perfect agreement with the concept of the QCD factorization. Applying the same analysis to the amplitudes $A_{q q}, A_{q g}, A_{g q}$, we arrive to the representation of the hadron amplitude $A_{h}$ in terms of the QCD factorization. Obviously, this factorization differs from the $k_{T^{-}}$and collinear factorization, so we again refer to it as the Basic Factorization. 


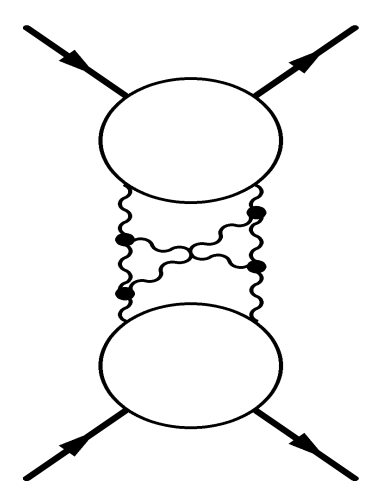

(a)

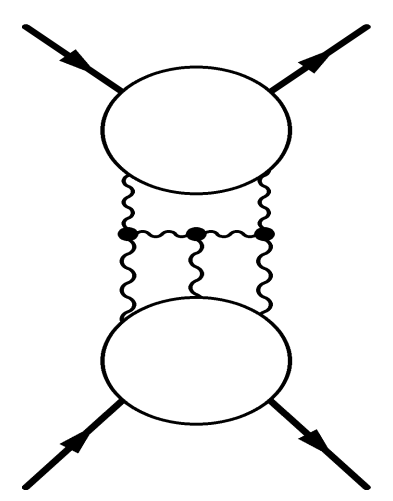

(b)

FIG. 9. Convolutions for $A_{h}$ beyond the Born approximation.

\section{A. Transition to the $k_{T}$-factorization}

The transition to $k_{T}$-factorization means performing the integrations over $\alpha_{1}$ and $\beta_{2}$ in Eqs. (25127) without dealing with the amplitude $\left(s^{\prime} / k^{2}\right) M$. This is possible only if these integrations are restricted as follows:

$$
s \alpha_{1} \beta_{1} \ll k_{1 \perp}^{2}, \quad s \alpha_{2} \beta_{2} \ll k_{2 \perp}^{2} .
$$

The meaning of these restrictions is that the virtualities of the intermediate partons are space-like. This agrees with the known fact that the virtualities of the ladder partons in the Leading Logarithmic Approximation are space-like.

Restriction (30) allows us to integrate over $\beta_{2}$ and $\alpha_{1}$ in Eqs. (25127), arriving at the following expression for $A_{h}(s, t)$ in $k_{T}$-factorization:

$$
A_{h}(s, t)=\int d \alpha_{2} d \beta_{1} d k_{1 \perp}^{2} d k_{2 \perp}^{2} \widetilde{\Psi}\left(s \alpha_{2}, k_{2 \perp}^{2}, k_{2 \perp}^{\prime 2}\right) \frac{1}{k_{b \perp}^{2}} A^{(p e r t)} \frac{1}{k_{a \perp}^{2}} \Psi\left(s \beta_{1}, k_{1 \perp}^{2}, k_{1 \perp}^{\prime 2}\right)
$$

with the perturbative amplitude $A^{(\text {pert })}$ and non-perturbative parton distributions $\Psi, \widetilde{\Psi}$ defined as follows:

$$
\begin{gathered}
A^{(\text {pert })}=\left(s^{\prime} / k_{\perp}^{2}\right) M\left(\ln \left(s^{\prime} / k_{1, \perp}^{2}\right), \ln \left(s^{\prime} / k_{\perp}^{2}\right), \ln \left(s^{\prime} / k_{1, \perp}^{\prime 2}\right), \ln \left(s^{\prime} / k_{\perp}^{\prime 2}\right)\right), \\
\widetilde{\Psi}=\int_{s_{1}^{(0)} / s}^{k_{2 \perp}^{2} / s \alpha_{2}} d \beta_{2} \widetilde{T}\left(s \beta_{2}, k_{2 \perp}^{2}, k_{2 \perp}^{\prime 2}\right), \quad \Psi=\int_{s_{2}^{(0)} / s}^{k_{1 \perp}^{2} / s \beta_{1}} d \alpha_{1} T\left(s \alpha_{1}, k_{1 \perp}^{2}, k_{1 \perp}^{\prime 2}\right)
\end{gathered}
$$

with $s_{1}^{(0)}$ and $s_{2}^{(0)}$ being the minimal invariant energy of the upper and lowest blobs, respectively (cf. Eq. (18)). Putting $t=0$, then taking the imaginary part of Eq. (31) and using the Optical theorem, we arrive at the expression for the total cross-section $\sigma_{t o t}$ of the hadron scattering in $k_{T}$-factorization:

$$
\sigma_{t o t}=\int d \alpha_{2} d \beta_{1} \frac{d k_{1 \perp}^{2}}{k_{1 \perp}^{2}} \frac{d k_{2 \perp}^{2}}{k_{2 \perp}^{2}} \widetilde{\Phi}\left(s \alpha_{2}, k_{2 \perp}^{2}\right) \sigma_{t o t}^{(p e r t)}\left(s^{\prime}, k_{1 \perp}^{2}, k_{2 \perp}^{2}\right) \Phi\left(s \beta_{1}, k_{1 \perp}^{2}\right)
$$

with the new parton distributions $\Phi\left(s \beta_{1}, k_{1 \perp}^{2}\right)$ and $\widetilde{\Phi}\left(s \alpha_{2}, k_{2 \perp}^{2}\right)$ defined quite similarly to the parton distributions in Eq. (17): 


$$
\Phi\left(s \beta_{1}, k_{1 \perp}^{2}\right)=\Im \Psi\left(s \beta_{1}, k_{1 \perp}^{2}\right), \quad \widetilde{\Phi}\left(s \alpha_{2}, k_{2 \perp}^{2}\right)=\Im \tilde{\Psi}\left(s \alpha_{2}, k_{2 \perp}^{2}\right) .
$$

In Eq. (34) we have used the notation $\sigma_{\text {tot }}^{(p e r t)}$ for the total cross-section of the perturbative sub-process describing the scattering of two partons. $k_{T}$-factorization was introduced in Ref. [3] to replace the collinear factorization in the case when the BFKL Pomeron [6] is used for the description of $\sigma_{\text {tot }}^{(p e r t)}$. Collinear factorization can be used when alternative methods are exploited to describe $\sigma_{\text {tot }}^{(\text {pert })}$.

\section{B. Transition to collinear factorization}

The transition from $k_{T}$-factorization in Eq. (34) to collinear factorization can be done, assuming a peaked $k_{\perp}-$ dependence of the parton distributions involved, $\Phi, \widetilde{\Phi}$. This dependence is depicted in Fig. 5. Once we accept this assumption, we can neglect the $k_{\perp}$-dependence of $\sigma_{\text {tot }}^{(p e r t)}$ and integrate only the parton distributions $\Phi, \widetilde{\Phi}$ over $k_{1,2 \perp}$. As a result, we arrive at

$$
\sigma_{t o t}=\sum_{k, l} \int d \alpha_{2} d \beta_{1} \widetilde{\varphi}_{k}\left(s \alpha_{2}, \mu_{l}^{2}\right) \sigma_{t o t}^{(p e r t)}\left(s^{\prime}, \mu_{k}^{2}\right) \varphi_{k}\left(s \beta_{1}, \mu_{k}^{2}\right)
$$

where

$$
\varphi_{k}\left(s \beta_{1}, \mu_{k}^{2}\right)=\int_{D_{k}} d k_{1 \perp}^{2} \Phi\left(s \beta_{1}, k_{1 \perp}^{2}\right), \quad \widetilde{\varphi}_{k}\left(s \alpha_{2}, \mu_{k}^{2}\right)=\int_{D_{k}} d k_{2 \perp}^{2} \widetilde{\Phi}\left(s \alpha_{2}, k_{1 \perp}^{2}\right)
$$

where $D_{k}$ is the region around the maximum of the parton distributions at $k_{1,2 \perp}^{2}=\mu_{k}^{2}$.

The transitions from the basic form of QCD factorization to $k_{T}$-factorization and to collinear factorization for the non-singlet component of $A_{h}$ can be done quite similarly. Obviously, Eq. (36) differs from the conventional expression

$$
\sigma_{t o t}=\int d \alpha_{2} d \beta_{1} \widetilde{\phi}_{k}\left(s \alpha_{2}, \mu^{2}\right) \sigma_{t o t}^{(p e r t)}\left(s^{\prime}, \mu^{2}\right) \phi\left(s \beta_{1}, \mu^{2}\right)
$$

where the parton distributions $\phi, \widetilde{\phi}$ include both perturbative and non-perturbative contributions.

\section{COMPARISON OF EQS. (20/34) TO THE CONVENTIONAL FORM OF COLLINEAR FACTORIZATION}

The reduction of the $k_{T}$-factorization to collinear factorization led us to Eq. (20) for the DIS structure functions and Eq. (36) for the total cross-section. Obviously, these expressions differ a lot from the expressions written in the conventional form Eq. (5138) of collinear factorization. Let us show how Eqs. (20)36) can be brought to the conventional form. First of all, notice that the collinear factorization in Eqs. (20]36) differs from the conventional form in the following aspects:

(A) There is only one factorization scale $\mu$ in Eqs. (538) and and this scale does not bear any physical meaning, so $\mu$ can be chosen arbitrary. On the contrary, Eqs. (20136) admit the possibility of having several intrinsic scales $\mu_{r}$, each corresponds to the maximum $k_{\perp}^{2}=\mu_{r}^{2}$ of the unintegrated parton distributions.

(B) The parton distributions $\varphi, \widetilde{\varphi}$ in Eqs. (20136) include the non-perturbative distributions only whereas the conventional form operates with the distributions $\phi, \widetilde{\phi}$ where there are both perturbative and non-perturbative contributions.

Despite such a considerable difference between these two approaches, it is easy to show that in fact Eqs. (20136) can be brought to the form of Eqs. (5138). This can be done identically for Eq. (20) and Eq. (36), so in what follows we will focus on considering Eq. (20). Obviously, Eq. (5) can be written as (cf. Eq. (22))

$$
f\left(x, Q^{2}\right)=E\left(Q^{2} / \mu^{2}\right) \otimes \tilde{\phi}\left(\mu^{2}\right)
$$


where $E\left(Q^{2} / \mu^{2}\right)$ denotes any of the appropriate evolution operator for performing the evolution from $\mu^{2}$ to $Q^{2}$. We have dropped its $x$-dependence as unessential. In particular, it can be the DGLAP -evolution operator [4]. Using such notation, Eq. (20) can be rewritten as follows:

$$
f\left(x, Q^{2}\right)=\sum_{k} E\left(Q^{2} / \mu_{k}^{2}\right) \otimes \phi_{k}\left(\mu_{k}^{2}\right)=E\left(Q^{2} / \mu^{2}\right) \otimes \sum_{k} E\left(\mu^{2} / \mu_{k}^{2}\right) \otimes \phi_{k}\left(\mu_{k}^{2}\right)=E\left(Q^{2} / \mu^{2}\right) \otimes \phi\left(\mu^{2}\right)
$$

with

$$
\phi\left(\mu^{2}\right)=\sum_{k} E\left(\mu^{2} / \mu_{k}^{2}\right) \otimes \varphi_{k}\left(\mu_{k}^{2}\right)
$$

Eq. (40) makes sense if $\mu>\mu_{k}$. This perfectly agrees with the actual situation: the scale $\mu$ in the standard approach is usually chosen $\sim 1 \mathrm{GeV}$ whereas the intrinsic scales $\mu_{k}$ are supposedly non-perturbative and they are therefore expected to be not far from $\Lambda_{Q C D}$. Eq. (40) demonstrates that the conventional approach and our approaches can be converted each into other when the factorization scale $\mu$ is chosen at the GeV range, so they are equivalent and indistinguishable when $\mu$ is kept within the GeV range or higher. In this case the parton distributions should explicitly include perturbative terms in addition to the non-perturbative ones. They become distinguishable at smaller values of the factorization scale, close to $\Lambda_{Q C D}$ (where the perturbative component vanishes), providing there are several (more than one) intrinsic scales $\mu_{k}$, otherwise they are again indistinguishable. However, the difference between them can be found in the framework of the $k_{T}$-factorization where the existence of the maximums in dependence $\Phi=\Phi\left(k_{\perp}^{2}\right)$ can be checked with analysis of experimental data.

\section{ULTRAVIOLET BEHAVIOR OF THE PARTON DISTRIBUTIONS $T_{S}, T_{N S}$}

The basic factorization convolutions for the Compton amplitudes (i.e. Eqs. (912) complemented by Eqs. (10)) and for the hadron amplitudes $A_{h}$ (i.e. Eqs. (2527) complemented by Eqs. (28)) are IR-stable. In addition, they should be UV-stable. The UV-stability of the Compton amplitudes was discussed in detail in Ref. [7]. It was proved that the convolutions are UV-stable when the parton distributions $T_{S}(\alpha), T_{N S}(\alpha)$ at large $|\alpha|$ behave as follows:

$$
T_{N S} \sim|\alpha|^{-1-h}, \quad T_{S} \sim|\alpha|^{-h},
$$

with $h>0$. It is easy to show that the singlet parton distributions $T\left(\alpha_{1}\right), \widetilde{T}\left(\beta_{2}\right)$ in the convolutions of Eqs. (25)27) should exhibit absolutely the same UV behavior:

$$
T\left(\alpha_{1}\right) \sim\left|\alpha_{1}\right|^{-h}, \quad \widetilde{T} \sim \beta_{2}^{-h}
$$

\section{RESTRICTIONS ON THE PARTON DISTRIBUTIONS}

We obtain below the restriction on the parton distributions in the collinear and $k_{T}$-factorizations that follow from the integrability of the basic convolutions. We exploit here the obvious mathematical requirement of stability of the convolutions in both the IR and UV -regions. These restrictions can also be used as suggestions when PDF fits are constructed.

\section{A. Parton distributions in $k_{T}$-factorization}

The fits for the parton distributions in $k_{T}$-factorization are commonly fixed from phenomenological considerations (see Ref. [11]). Below we give some restrictions on the fits following from theoretical grounds. The parton distributions $\Phi$ in $k_{T}$-factorization are defined in Eq. (17) (see also Eq. (35) ) as the integrals of the totally unintegrated parton densities. Combining this equation with the restriction of Eq. (10) (see also Eq. (28)) on their IR-behavior leads us to the most general form of $\Phi_{N S}, \Phi_{S}$ :

$$
\begin{aligned}
\Phi_{N S} & =\left(k_{\perp}^{2}\right)^{\gamma} \chi_{N S}^{(1)}\left(\beta, k_{\perp}^{2}\right)+\left(k_{\perp}^{2}\right)^{\gamma-h} \beta^{h} \chi_{N S}^{(2)}\left(\beta, k_{\perp}^{2}\right), \\
\Phi_{S} & =\left(k_{\perp}^{2}\right)^{1+\gamma} \chi_{S}^{(1)}\left(\beta, k_{\perp}^{2}\right)+\left(k_{\perp}^{2}\right)^{2+\gamma-h} \beta^{-1+h} \chi_{S}^{(2)}\left(\beta, k_{\perp}^{2}\right),
\end{aligned}
$$


with the requirement

$$
\gamma-h>0
$$

We remind that the positive parameters $\gamma$ and $h$ were introduced in Eqs. (10142) for the Compton amplitudes to guarantee their IR and UV stability, respectively. When the Compton amplitudes are considered in basic factorization, $\gamma$ and $h$ are independent. However, they proved to be related by Eq. (45) when basic factorization has been reduced to $k_{T}$-factorization: Eq. (45) ensures the IR-stability of the structure functions in $k_{T}$-factorization. Eq. (44) shows that the parton densities in $k_{T}$-factorization consist of two terms, each involves different power factors of $k_{\perp}^{2}$ and the last $k_{\perp}^{2}$-factor involves the longitudinal variable $\beta$. In addition, they involve arbitrary functions $\chi_{S, N S}^{(1,2)}$. All $\chi_{S, N S}^{(1,2)} \rightarrow$ const at $k_{\perp}^{2} \rightarrow 0$. $\Phi_{S, N S}$ originate from $T_{S, N S}$, so the requirements of the UV stability in Eqs. (42) 43) mean that the fits for $\Phi_{N S}$ should not contain the factors $\beta^{-a}$, with $a>0$, whereas the requirement on fits for $\Phi_{S}$ is less severe: they can contain such factors, providing $a<1$. However, sometimes the same parton distributions contribute to the singlet and non-singlet constructions. For example, this is true for the flavor singlet components of the structure functions $F_{1}$ (addressed as the singlet in the present paper) and $F_{2}$ (addressed as the non-singlet): they both involve the same parton distributions. In such cases, the singlet fits should obey the more severe requirement for the non-singlets.

In order to make possible the transitions to the collinear factorization, the $k_{\perp}$-dependence of $\chi_{S, N S}^{(1,2)}$ should be of a peaked (for example, Gaussian) form.

\section{B. Parton distributions in collinear factorization}

The conventional fits 12] for the parton distributions in collinear factorization are also constructed from purely phenomenological considerations. Below we impose some restrictions on these fits arising from integrability the factorization convolutions. The standard fits for the initial parton densities $\delta q, \delta g$ in collinear factorization are known to include a normalization $N$, the singular factors $x^{-a}$, with $a>0$, and regular terms. For example,

$$
\delta q=N x^{-a}(1-x)^{b}\left(1+c x^{d}\right),
$$

where the parameters $N, a, b, c, d$ are positive. They are specified from analysis of experimental data. Although such expressions do not look explicitly like the ones obtained with the perturbative methods, nonetheless there are two options to study:

(i) The singular factors $x^{-a}$ appear as a result of total resummations of the perturbative contributions. In this case we identify the fits with the parton distributions $\phi$ containing both perturbative and non-perturbative contributions. The factors $x^{-a}$ in such distributions mimic the resummation of either the leading logarithms (see [10]) or sub-leading logarithms (see [2]). In the both cases the singular factors can be removed from the fits when the resummation is accounted for because the resummation of logarithms of $x$ can be absorbed by the coefficient functions.

(ii) The whole fits Eq. (46), including the singular factors $x^{-a}$, have the non-perturbative origin. In this case we identify the fits in Eq. (46) with the non-perturbative distributions $\varphi$ defined in Eqs. (21137) and apply to them the restrictions of Eqs. (4243). These restrictions exclude the use of the singular factors in the expressions for the non-singlet structure functions $F_{2}, F_{1}^{N S}, g_{1}$, etc and also suppress the singular factors with $a>1$ in the expressions for the singlet $F_{1}$. However, the parton distributions used for $F_{1}$ and $F_{2}$ are identical, therefore the suppression of the singular factors with $a>0$ can be applied to all structure functions, including the singlet $F_{1}$. The singular factors $x^{-a}$ in the DGLAP fits for initial parton densities should be removed from the fits because they contradict to the integrability of the basic convolutions of the Compton amplitudes. Let us remark that the removal of the factors $x^{-a}$ from the fits and replacing such factors by the total resummation of the logarithms can reduce the fits in Eq. (46) down to much simpler expressions like $N(1-x)^{b}$ or, when the starting values of $x$ are small, down to constants.

\section{SUMMARY}

We have shown that the scattering amplitudes of the processes Eq. (10) and Eq. (31) can be represented as basic convolutions where the perturbative and non-perturbative contributions are separated (i.e. located in different blobs). The basic convolutions correspond to concept of QCD factorization but they are more general than collinear and $k_{T}$-factorization. The perturbative components of the basic convolutions are off-shell and therefore they are not gauge-invariant. However, their gauge invariance is restored at high energy (small $x$ ). When the virtualities of the 
intermediate partons are space-like, the basic factorization can be approximately reduced to $k_{T}$-factorization. In its turn, $k_{T}$-factorization can approximately be reduced to collinear factorization if the peaked dependence on $k_{\perp}$ (see Fig. 5) is assumed for the unintegrated parton distributions and the parton distributions are altogether nonperturbative. The sharper the peaks in Fig. 5 are, the higher is the accuracy of the reduction. We stress that this hypothesis can be checked by analysis of experimental data. In contrast to the conventional scenario of collinear factorization, we have arrived at the more involved form of collinear factorization, with one of several (intrinsic) scales $\mu_{r}$ corresponding to the maxima of the unintegrated parton distributions. Using perturbative evolution to increase the scale, we have brought this complicated many-scale picture to the conventional form, where only one arbitrary scale is used explicitly and the parton distributions include both perturbative and non-perturbative contributions. The integrations in the basic convolutions run over the whole phase space but they must yield finite results. Exploiting this obvious requirement allows us to obtain restrictions on the parton distributions in both collinear and $k_{T}$-factorization. We have shown that the parton distributions in $k_{T}$-factorization should consist of two terms, with different powers of $k_{\perp}^{2}$. Then, we exclude the use of factors $x^{-a}$ from the fits for the parton distributions both in the collinear and $k_{T}$-factorization. Finally, let us remark that the results obtained in the present paper can easily be extended to inelastic hadron scattering at high energy.

\section{ACKNOWLEDGMENTS}

We are grateful to B. Webber for interesting discussions. We are also grateful to S. Alekhin and G. Lykasov for useful correspondence. The work is partly supported by Grant RAS 9C237, Russian State Grant for Scientific School RSGSS-65751.2010.2 and EU Marie-Curie Research Training Network under contract MRTN-CT-2006-035505 (HEPTOOLS).

\section{Appendix A: Gauge invariance of the Compton scattering amplitude}

\section{Notation for Compton scattering}

Let us consider the Compton scattering

$$
\gamma(q)+q(p) \rightarrow \gamma\left(q^{\prime}\right)+q\left(p^{\prime}\right)
$$

We denote by $A_{\mu \nu}$ the scattering amplitude of the process (A1). Gauge invariance states that

$$
q_{\mu} A_{\mu \nu}=q_{\nu} A_{\mu \nu}=0
$$

\section{Compton scattering amplitude in the Born approximation}

In the Born approximation the scattering amplitude $A_{\mu \nu}$ is represented by the graphs depicted in Fig. 3.

$$
A_{\mu \nu}=\frac{<p^{\prime}\left|\gamma_{\nu}(\hat{p}+\hat{q}+m) \gamma_{\mu}\right| p>}{(p+q)^{2}-m^{2}}+\frac{<p^{\prime}\left|\gamma_{\mu}\left(\hat{p}^{\prime}-\hat{q}+m\right) \gamma_{\nu}\right| p>}{\left(p^{\prime}-q\right)^{2}-m^{2}}
$$

\section{Gauge invariance of the Compton amplitude in arbitrary kinematics}

It follows from Eq. (A3) that

$$
\begin{aligned}
q_{\mu} A_{\mu \nu} & =-\frac{<p^{\prime}\left|\gamma_{\nu} \hat{q}(\hat{p}-m)\right| p>}{(p+q)^{2}-m^{2}}-\frac{<p^{\prime}\left|\left(\hat{p}^{\prime}-m\right) \hat{q} \gamma_{\nu}\right| p>}{\left(p^{\prime}-q\right)^{2}-m^{2}} \\
& +<p^{\prime}\left|\gamma_{\nu}\right| p>\left[\frac{q^{2}+2 p q}{(p+q)^{2}-m^{2}}-\frac{q^{2}-2 p^{\prime} q}{\left(p^{\prime}-q\right)^{2}-m^{2}}\right]
\end{aligned}
$$


Eq. (A4) can be re-written as follows:

$$
\begin{aligned}
q_{\mu} A_{\mu \nu} & =-\frac{<p^{\prime}\left|\gamma_{\nu} \hat{q}(\hat{p}-m)\right| p>}{(p+q)^{2}-m^{2}}-\frac{<p^{\prime}\left|\left(\hat{p}^{\prime}-m\right) \hat{q} \gamma_{\nu}\right| p>}{\left(p^{\prime}-q\right)^{2}-m^{2}} \\
& -<p^{\prime}\left|\gamma_{\nu}\right| p>\left[\frac{p^{2}-m^{2}}{(p+q)^{2}-m^{2}}-\frac{p^{\prime 2}-m^{2}}{\left(p^{\prime}-q\right)^{2}-m^{2}}\right]
\end{aligned}
$$

Obviously, Eq. (A5) yields a zero result only when the incoming and outgoing quarks are on-shell. In contrast, the photons can be either on-shell or off-shell.

Let us focus on considering forward Compton scattering.

\section{Forward Compton amplitude in the Born approximation}

In this case

$$
A_{\mu \nu}=\frac{<p\left|\gamma_{\nu}(\hat{p}+\hat{q}+m) \gamma_{\mu}\right| p>}{(p+q)^{2}-m^{2}}+\frac{<p\left|\gamma_{\mu}(\hat{p}-\hat{q}+m) \gamma_{\nu}\right| p>}{(p-q)^{2}-m^{2}} .
$$

Obviously, the forward Compton amplitude is gauge-invariant when the quark is on-shell. However, now we allow it to be off-shell. In order to study gauge invariance for an off-shell quark, we rewrite $A_{\mu \nu}$ as follows:

$$
A_{\mu \nu}=A_{\mu \nu}^{(1)}+A_{\mu \nu}^{(2)}+A_{\mu \nu}^{(3)}
$$

where

$$
\begin{aligned}
& A_{\mu \nu}^{(1)}=\left[\frac{<p\left|\gamma_{\nu} \hat{p} \gamma_{\mu}\right| p>}{(p+q)^{2}-m^{2}}+\frac{<p\left|\gamma_{\mu} \hat{p} \gamma_{\nu}\right| p>}{(p-q)^{2}-m^{2}}\right], \\
& A_{\mu \nu}^{(2)}=\left[\frac{<p\left|\gamma_{\nu} \hat{q} \gamma_{\mu}\right| p>}{(p+q)^{2}-m^{2}}-\frac{<p\left|\gamma_{\mu} \hat{q} \gamma_{\nu}\right| p>}{(p-q)^{2}-m^{2}}\right], \\
& A_{\mu \nu}^{(3)}=m\left[\frac{<p\left|\gamma_{\nu} \gamma_{\mu}\right| p>}{(p+q)^{2}-m^{2}}+\frac{<p\left|\gamma_{\mu} \gamma_{\nu}\right| p>}{(p-q)^{2}-m^{2}}\right]
\end{aligned}
$$

Let us multiply Eq. (A7) by $q_{\nu}$ and represent the result as follows:

$$
G_{\mu} \equiv q_{\nu} A_{\mu \nu}=G_{\mu}^{(1)}+G_{\mu}^{(2)}+G_{\mu}^{(3)},
$$

with

$$
\begin{gathered}
G_{\mu}^{(1)} \equiv q_{\nu} A_{\mu \nu}^{(1)}=\left[\frac{<p\left|\hat{q} \hat{p} \gamma_{\mu}\right| p>}{(p+q)^{2}-m^{2}}+\frac{<p\left|\gamma_{\mu} \hat{p} \hat{q}\right| p>}{(p-q)^{2}-m^{2}}\right], \\
G_{\mu}^{(2)} \equiv q_{\nu} A_{\mu \nu}^{(2)}=q^{2}<p\left|\gamma_{\mu}\right| p>\left[\frac{1}{(p+q)^{2}-m^{2}}-\frac{1}{(p-q)^{2}-m^{2}}\right], \\
G_{\mu}^{(3)} \equiv q_{\nu} A_{\mu \nu}^{(3)}=m\left[\frac{<p\left|\hat{q} \gamma_{\mu}\right| p>}{(p+q)^{2}-m^{2}}+\frac{<p\left|\gamma_{\mu} \hat{q}\right| p>}{(p-q)^{2}-m^{2}}\right] .
\end{gathered}
$$

Now let us simplify the Dirac structures in (A10):

$$
\begin{aligned}
& \gamma_{\mu} \hat{q} \hat{p}=-\imath \epsilon_{\mu \lambda \rho \sigma} q_{\lambda} p_{\rho} \gamma_{5} \gamma_{\sigma}+q_{\mu} \hat{p}-p_{\mu} \hat{q}+p q \gamma_{\mu} \\
& \hat{q} \hat{p} \gamma_{\mu}=\imath \epsilon_{\mu \lambda \rho \sigma} q_{\lambda} p_{\rho} \gamma_{5} \gamma_{\sigma}+q_{\mu} \hat{p}-p_{\mu} \hat{q}+p q \gamma_{\mu}
\end{aligned}
$$


This makes it possible to represent $G_{\mu}^{(1)}$ as a sum of symmetrical and antisymmetrical contributions:

$$
G_{\mu}^{(1)}=G_{\mu}^{(1 A)+} G_{\mu}^{(1 S)}
$$

with

$$
G_{\mu}^{(1 A)}=\imath \epsilon_{\mu \lambda \rho \sigma} q_{\lambda} p_{\rho} \frac{<p\left|\gamma_{5} \gamma_{\sigma}\right| p>}{w}\left[\frac{1}{1-x-z}+\frac{1}{1+x+z}\right]
$$

where $x=-q^{2} / w, z=m^{2} / w$ and $w=2 p q$.

Similarly,

$$
G_{\mu}^{(1 S)}=\frac{\left[q_{\mu}<p|\hat{p}| p>-p_{\mu}<p|\hat{q}| p>+p q<p\left|\gamma_{\mu}\right| p>\right]}{w}\left[\frac{1}{1-x-z}-\frac{1}{1+x+z}\right] .
$$

In order to retain the CP-invariance in (A15), the term $<p\left|\gamma_{5} \gamma_{\sigma}\right| p>$ must yield the spin contribution $\sim S_{\sigma}$. This converts the antisymmetrical factor in A15) into

$$
\epsilon_{\mu \lambda \rho \sigma} q_{\lambda} p_{\rho} S_{\sigma} .
$$

In order to simplify (A16) we use the approximation

$$
<p\left|\gamma_{\mu}\right| p>\approx p_{\mu},
$$

which allows us to re-write (A16) as

$$
G_{\mu}^{(1 S)} \approx\left[q_{\mu} p^{2}-p_{\mu} p q+p_{\mu} p q\right] \frac{<p \mid p>}{w}\left[\frac{1}{1-x-z}-\frac{1}{1+x+z}\right]
$$

Obviously, the expressions on the r.h.s. of Eqs. A15 A19) are not equal to zero, which means that gauge invariance for $A_{\mu \nu}^{(1)}$ is broken. The same is true for $A_{\mu \nu}^{(2)}$ and $A_{\mu \nu}^{(3)}$ when the quark is off-shell.

\section{Restoration of the gauge invariance for forward Compton scattering at small $x$}

In the high-energy limit where $w \gg\left|q^{2}\right|,\left|\left(p^{2}-m^{2}\right)\right|$, i.e. where both

$$
x \ll 1
$$

and

$$
z \ll 1
$$

the expression in the square brackets in Eq. (A19) becomes small and therefore in this kinematics $G_{\mu}^{(1 S)} \sim \max [x, z] \approx$ 0 . Then, in this kinematic region the spin $S_{\rho}$ becomes mostly longitudinal, so the expression in (A17) is nearly zero and therefore $G_{\mu}^{(1 A)} \approx 0$. Eq. (A11) demonstrates that $G_{\mu}^{(2)}$ in region (A20) is proportional to $x$, so $G_{\mu}^{(2)} \approx 0$. Finally, let us notice that $G_{\mu}^{(3)} \sim m$, so it becomes small in the small-x region (A20). Therefore, the gauge invariance of the forward Compton amplitude, although broken when the off-shell quark is off-shell, is restored in the limit of small $x$ and $z$ for the unpolarized Compton scattering amplitudes and for those spin-dependent amplitudes, in which the spin is longitudinal. 


\section{Accounting for radiative corrections}

The most important radiative corrections in the small- $x$ region are logarithmic ones. The arguments of the logarithms can be chosen as $w / p^{2}$ and $Q^{2} / p^{2}$. Accounting for the radiative corrections with logarithmic accuracy does not destroy the Born structure of the forward Compton amplitude. Then, it is obvious that $\ln \left(w / p^{2}\right)$ and $\ln \left(Q^{2} / p^{2}\right)$ can be large only if $p^{2} \ll Q^{2}, w$. So, the main contributions in the small- $x$ region come from quark virtualities obeying (A21). Therefore the total resummation of such contributions does not break the gauge invariance of the Compton amplitude at small $x$. This gives us the right to consider the factorization convolutions with the two-parton intermediate states only and to neglect all other intermediate states with greater numbers of partons without breaking gauge invariance at small $x$.

\section{Appendix B: Gauge invariance for quark-quark scattering in collinear kinematics}

Let us consider the quark-quark scattering

$$
q\left(p_{1}\right)+q\left(p_{2}\right) \rightarrow q\left(p_{1}^{\prime}\right)+q\left(p_{2}^{\prime}\right)
$$

in collinear kinematics where $p_{1} \approx p_{1}^{\prime}, p_{2} \approx p_{2}^{\prime}$. In what follows we will focus on the colorless, in the $t$-channel, part of the scattering amplitude of this process. In other words, we will consider the amplitude with vacuum quantum numbers in the $t$-channel. In the lowest-order approximation the colorless part of the scattering amplitude $A_{0}$ is depicted in Fig. 10.

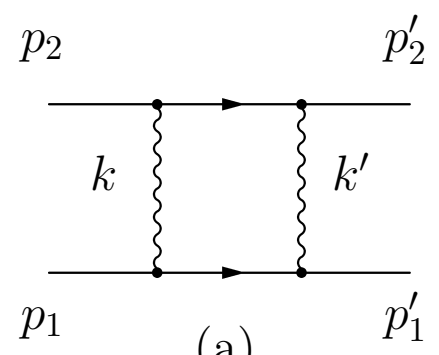

(a)

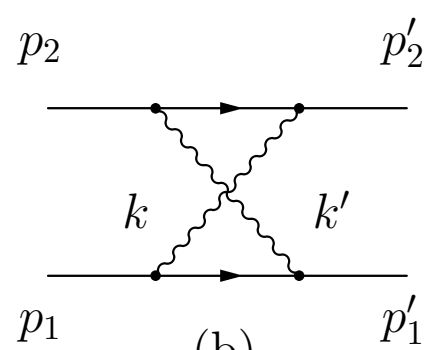

(b)

FIG. 10. Colorless contribution to the quark scattering in the lowest order.

We write it as follows:

$$
A_{0}=A_{s}+A_{u}
$$

where

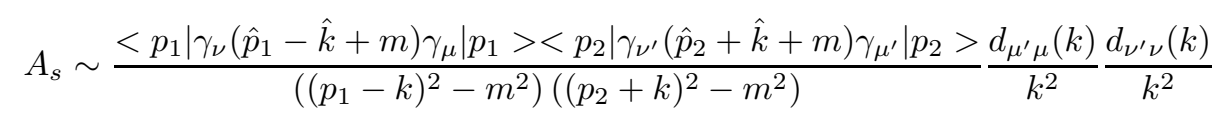

and

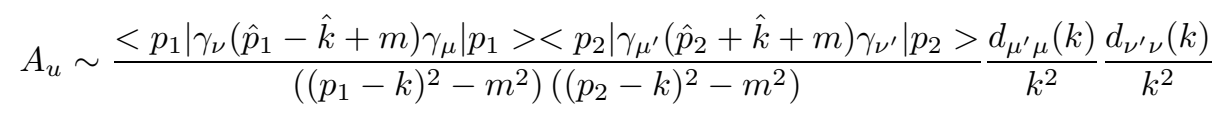

As our object is to study the gauge invariance of $A_{0}$, we have omitted in Eqs. (B3B4) all factors unessential for that. As is well-known, the leading contributions to $A_{s}$ and $A_{u}$ come from the terms $\sim p_{1}, p_{2}$ in the numerators of $B 3$, (B4), with $k$ and $m$ neglected. The remaining structures are obviously symmetrical under the replacements $\mu \rightleftharpoons \nu$ and $\mu^{\prime} \rightleftharpoons \nu^{\prime}$. Therefore we can write them as 


$$
A_{s}^{L}=\frac{S_{\mu \nu}\left(p_{1}\right) S_{\mu^{\prime} \nu^{\prime}}\left(p_{2}\right)}{\left(k^{2}-2 p_{1} k+\left(p_{1}^{2}-m^{2}\right)\right)\left(k^{2}+2 p_{2} k+\left(p_{2}^{2}-m^{2}\right)\right)} \frac{d_{\mu^{\prime} \mu}(k)}{k^{2}} \frac{d_{\nu^{\prime} \nu}(k)}{k^{2}}
$$

and

$$
A_{u}^{L}=\frac{S_{\mu \nu}\left(p_{1}\right) S_{\mu^{\prime} \nu^{\prime}}\left(p_{2}\right)}{\left(\left(k^{2}-2 p_{1} k+\left(p_{1}^{2}-m^{2}\right)\right)\left(k^{2}-2 p_{2} k+\left(p_{2}^{2}-m^{2}\right)\right)\right.} \frac{d_{\mu^{\prime} \mu}(k)}{k^{2}} \frac{d_{\nu^{\prime} \nu}(k)}{k^{2}}
$$

with

$$
S_{\mu \nu}\left(p_{1}\right)=<p_{1}\left|\gamma_{\nu} \hat{p}_{1} \gamma_{\mu}\right| p_{1}>, S_{\mu^{\prime} \nu^{\prime}}\left(p_{1}\right)=<p_{2}\left|\gamma_{\mu^{\prime}} \hat{p}_{2} \gamma_{\nu^{\prime}}\right| p_{2}>.
$$

Gauge invariance means that the replacement of $d_{\nu^{\prime} \nu}(k)$ by $k_{\nu} \phi_{\nu^{\prime}}(k), \phi$ being an arbitrary function, yields a zero result. Multiplying $S_{\mu \nu}\left(p_{1}\right)$ by $k_{\nu}$ and substituting the result in Eqs. (B5]B6) proves the breaking of gauge invariance. However, in the high-energy limit where

$$
\left|2 p_{1} k\right|,\left|2 p_{2} k\right| \gg\left|k^{2}\right|,\left|\left(p^{2}-m^{2}\right)\right|
$$

gauge invariance is restored. This is the appropriate region to produce the leading logarithmic contribution. This means that accounting for these contributions does not destroy the gauge invariance of the amplitude $A_{0}$.

[1] D. Amati, R. Petronzio, G. Veneziano. Nucl. Phys. B 140 (1978) 54; A.V. Efremov, A.V. Radyushkin. Teor.Mat.Fiz. 42 (1980) 147; Theor.Math.Phys.44 (1980)573; Teor.Mat.Fiz.44 (1980)17; Phys.Lett.B63 (1976) 449; Lett.Nuovo Cim.19 (1977)83; S. Libby, G. Sterman. Phys. Rev. D18 (1978) 3252. S.J. Brodsky and G.P. Lepage. Phys. Lett. B 87 (1979) 359; Phys. Rev. D 22 (1980) 2157; J.C. Collins and D.E. Soper. Nucl. Phys.B 193 (1981) 381; J.C. Collins and D.E. Soper. Nucl. Phys.B 194 (1982) 445; J.C. Collins, D.E. Soper and G. Sterman. Nucl. Phys.B 250 (1985) 199.

[2] A.V. Efremov and I.F. Ginzburg. Fortsch.Phys.22 (1974) 575; A.V. Efremov and A.V. Radyushkin. Report JINR E2-80-521; Mod.Phys.Lett. A24 (2009) 2803.

[3] S. Catani, M. Ciafaloni, F. Hautmann. Phys. Lett. B 242 (1990) 97; Nucl.Phys.B366 (1991) 135.

[4] G. Altarelli and G. Parisi, Nucl. Phys.B126 (1977) 297; V.N. Gribov and L.N. Lipatov, Sov. J. Nucl. Phys. 15 (1972) 438 ; L.N.Lipatov, Sov. J. Nucl. Phys. 20 (1972) 95; Yu.L. Dokshitzer, Sov. Phys. JETP 46 (1977) 641.

[5] B.I. Ermolaev, M. Greco, S.I. Troyan. Acta Phys.Polon.B38 (2007) 2243.

[6] E.A. Kuraev, L.N. Lipatov and V.S. Fadin, Sov. Phys. JETP 44, 443 (1976); E.A. Kuraev, L.N. Lipatov and V.S. Fadin, Sov. Phys. JETP 45, 199 (1977); I.I. Balitsky and L.N. Lipatov, Sov. J. Nucl. Phys. 28, 822 (1978).

[7] B.I. Ermolaev, M. Greco, S.I. Troyan. Eur.Phys.J.C71 (2011) 1750; arXiv:1111.0556

[8] V.V. Sudakov. Sov. Phys. JETP 3(1956)65.

[9] J.C. Collins, T.C. Rogers, A.M. Stasto. Phys.Rev.D77 (2008) 085009.

[10] B.I. Ermolaev, M. Greco, S.I. Troyan, Phys.Lett.B622 93 (2005) 101; Riv.Nuovo Cim.33 (2010) 57.

[11] B. Andersson, et al. Eur. Phys. J. C 25, 77 (2002). K. Golec-Biernat, M. Wusthoff. Phys.Rev. D60 (1999) 114023; Jung H., Proc. of the DIS 2004, Strbaske Pleso, Slovakia, arXiv:0411287; A.V. Kotikov, A.V. Lipatov, N.P. Zotov. Eur. Phys. J. C 27, 219 (2003); G. Watt, A.D. Martin and M.G. Ryskin. Eur.Phys.J.C.31 (2003) 73; Phys.Rev. D, 70 (2004) 014012; Erratum: ibid. 70 (2004) 079902; N.I. Kochelev. Phys.Lett.,B, 426 (1998) 149; I.P. Ivanov, N.N. Nikolaev. Phys.Rev.D 65 (2002) 054004; G.I. Lykasov, V.A. Bednyakov, A.A. Grinyuk, M. Poghosyan, A.G. Dolbilov. arXiv:1109.1469

[12] G. Altarelli, R.D. Ball, S. Forte, G. Ridolfi. Nucl.Phys.B496 (1997)337; R. D. Ball, L. Del Debbio, S. Forte, A. Guffanti, J. I. Latorre, J. Rojo and M. Ubiali. Nucl. Phys.B838 (2010) 136; E. Leader, A.V. Sidorov, D.B. Stamenov. Phys.Rev.D73 (2006)034023; J. Blumlein, H. Botcher. Nucl.Phys.B636 (2002)225; S. Alekhin, J. Blumlein, S. Klein and S. Moch. Phys.Rev.D81 (2010) 014032; A. D. Martin, W. J. Stirling, R. S. Thorne and G. Watt. Eur.Phys. J. C63 (2009) 189; H. L. Lai, M. Guzzi, J. Huston, Z. Li, P. M. Nadolsky, J. Pumplin and C. P. Yuan. arXiv:1007.2241; P. Jimenez-Delgado and E. Reya. Phys.Rev.D79 (2009) 074023; 